\title{
The Loop-Mediated Isothermal Amplification Method Instead of PCR in Detection Goals
}

\author{
Peyman Avakh Majelan, ${ }^{1}$ Mehdi Fatahi Bafghi, ${ }^{2}$ Javad Alizadeh, ${ }^{3}$ Nasrin Pourramezan, ${ }^{1}$ and Davoud \\ Afshar $^{4, *}$ \\ ${ }^{1}$ Department of Pathobiology, Faculty of Public Health, Tehran University of Medical Sciences, Tehran, IR Iran \\ ${ }^{2}$ Department of Microbiology, Faculty of Medicine, Shahid Sadoughi University of Medical Sciences, Yazd, IR Iran \\ ${ }^{3}$ Department of Human Anatomy and Cell Science, Faculty of Health Sciences, University of Manitoba, Winnipeg, Canada \\ ${ }^{4}$ Department of Microbiology, Zanjan University of Medical Sciences, Zanjan, IR Iran \\ "Corresponding author: Davoud Afshar, Department of Microbiology, Zanjan University of Medical Sciences, Zanjan, IR Iran. E-mail: davoodafshar@yahoo.com
}

Received 2015 February 3; Accepted 2015 May 25.

Keywords: Loop-Mediated, PCR, Detection

\section{Dear editor,}

Loop-mediated isothermal amplification (LAMP) was recently developed by Notomi et al. [1]. LAMP requires a DNA polymerase known as Bst polymerase (Bacillus stearothermophilus) which has strand displacement activity. As, this special feature distinguishes LAMP assay from other commonly used molecular methods such as PCR (polymerase chain reaction) so there is no need for advanced equipment such as thermal cyclers [2]. Unlike PCR, a set of 6 (or 4) primers (F3, B3, FIP, BIP, LF and LB) is needed for LAMP. Using 6 primers is much desirable and leads to more accurate results; however, design such primers could be challenging. In fact, in most cases, software is not be able to design two loop primers whereas primers simply can be designed manually. Primer Explorer (http://primerexplorer.jp/e) is commonly used software to design LAMP primers. The molecular basis of LAMP is based on turbidity due to the production of $\mathrm{Mg}_{2} \mathrm{P}_{2} \mathrm{O}_{7}$ [3]. This is considered to be a critical step in LAMP since the set-up process can be challengeable. Recently, utilization of SYBR green dye has made this step comfortable. These statements are striking properties but the question that has been remained unanswered is "Why the LAMP assay has not been expended unlike PCR?" The LAMP assay has been used to detect the pathogenic bacteria such as Nocardia seriolae, Pseudomonas putida, Streptococcus iniae, Staphylococcus aureus, Vibrio spp., Campylobacter jejuni, Yersinia enterocolitica and several other viruses. However, unlike PCR assay, there are not many LAMP-based commercial kits to specifically detect a given bacterium or virus, which may resulted from the complexity of LAMP. That being said, the PCR assay is both simple and user-friendly compared to LAMP. Moreover, sequencing can be regarded as an efficient method beside PCR as it confirms the accuracy of amplicon. Gen- erally, the simplicity of an assay can influence on its application. From another point of view, understanding of the LAMP mechanism is more difficult for most researchers. Hence, its application is not favorable and no one expect it to be a common assay in biological laboratories. The important step in LAMP assay is its last step in which changes in the color occur. It is believed that a basic modification such as using a dye like SYBR green is required and this would help improve LAMP assay. Thus, applying this modification in LAMP principle might promote its efficiency. It is hoped that by applying new modifications, it will be satisfactory in near future due to its high sensitivity and specificity.

\section{References}

1. Notomi T, Okayama H, Masubuchi H, Yonekawa T, Watanabe K, Amino $\mathrm{N}$, et al. Loop-mediated isothermal amplification of DNA. Nucleic Acids Res. 2000;28(12):E63. [PubMed: 10871386].

2. Yoshida A, Nagashima S, Ansai T, Tachibana M, Kato H, Watari H, et al. Loop-mediated isothermal amplification method for rapid detection of the periodontopathic bacteria Porphyromonas gingivalis, Tannerella forsythia, and Treponema denticola. J Clin Microbiol. 2005;43(5):2418-24. doi: 10.1128/JCM.43.5.2418-2424.2005. [PubMed: 15872275].

3. Mori Y, Nagamine K, Tomita N, Notomi T. Detection of loop-mediated isothermal amplification reaction by turbidity derived from magnesium pyrophosphate formation. Biochem Biophys Res Commun. 2001;289(1):150-4. doi: 10.1006/bbrc.2001.5921. [PubMed: 11708792]. 